Comme le couple est optique, que le mouvement propre de l'étoile $(A)$ n'est pas insignifiant et que l'étoile $(B)$ est très-proche (optiquement) je crois que des observations dans le but d'avoir la paraliaxe de $(A)$ ne seraient pas perdues. Il $y$ a, à la distance de $3^{\prime}$ à $4^{\prime}$ dans les angles de position $95^{\circ}, 130^{0}, 200^{0}$ trois étoiles de grandeur IO-I I qui disparaissent totalement dans le champ de ma lunette quand on introduit le plus faible reflet de lumière, qui n'est pas encore suffisant à laisser aper- cevoir les fils. D'ailleurs les mesures avec les fils illuminés ne sont pas susceptibles d'une exactitude satisfaisante. J'invite donc les astronomes qui disposent d'un équatorial capable de donner les mesures concernant ces petites étoiles, à faire les observations pour la parallaxe que je ne peux pas faire et qui seraient insuffsantes si on prenait pour étoile de comparaison seulement l'étoile $(B)$. Capodimonte, Juin I 881 .

\title{
Nachweis eines Fehlers in der Mondcharte.
}

\section{Von $\mathscr{F} . F . \mathscr{F}$ ul. Schmidt.}

Lohrmann zeichnet in Sect. 19 seiner Charte, in $54^{0}$ östl. Länge und - $7^{0} 5$ Breite, einen starken, 2-3 Meilen grossen Crater Nr. 20, der in der dargestellten Art die ähulichen Crater Nr. 4, 6, I 5 in der Nähe des Flamsteed an Augenfälligkeit ubertreffen muisste. An demselben Orte hat Mädler in seiner Charte (wenn man sehr genau die allzufeine Zeichnung ansieht), zwei pa. rallele schwache und kurze Hügel, von $N-S$ gerichtet. Bei Schröter könnte der Crater nur in Bd. I. Tab. 39 gesucht werden, wo man Billy und Hansteen dargestellt findet. Doch ist an dieser Stelle kein Detail zu erwarten. In Neisons Charte ist die fragliche Stelle leer.

Auf meiner Mondcharte, ebenfalls in Sect. 19, ist nun am angezeigten Orte ein grosser Crater Nr.3 Melloni gezeichnet, und nördlich von ihm ein schwaches hufeisenförmiges, gegen Norden offenes Gebilde, ähnlich einem sehr flachen Halbcrater, das ich $H$ nennen werde.

Um die Sachlage kurz zu erörtern, will ich zunächst bemerken, dass der starke Crater Nr. 20 bei Lohrmann, - Nr. 3 bei mir, oder Melloni, in den letzten 40 Jahren als solcher wahrscheinlich $z u$ keiner Zeit vorhanden war; dass aber an seinem Orte (bei Lohrmann), oder etwas nördlich von meinem $\mathrm{Nr} . \mathfrak{3}$, die schwache Figur $H$ anzunchmen sei.

Um zu einer strengen Entscheidung zu gelangen, genügte es mir nicht, allein die Cataloge zu meinen Originalzeichnungen $z u$ befragen, und demnach die angezeigten Abbildungen aufzusuchen. Ich entschloss mich, die ganze Reihe von mehr als 2900 Aufnahmen einzeln durchzusehen, und fand demnach folgende Resultate:

1842 Jan. 23. In meiner Phasenzeichnung Nr. 68 findet sich die Stelle leer. Bis Juni beob. ich nur an sehr schwachem Fernrohr.

1842 Febr. 22. In Phase Nr. 76 ist ein kleiner Crater gezeichnet.

1842 Dec. 14. Am Orte des Melloni ein kleiner Crater gezeichnet. Diese 3 Angaben sind jedoch ohne Gewicht.

In den Jahren I 843 bis I 865 fellt jede Beobachtung über diese Gegend, und auch in den handschriftlichen Notizen findet sich Nichts.
I 865 April 8. (Athen). 6 fuss. Refractor. Lichtgränze in $56^{\circ}$ Ost Länge. Abbd. $\mathrm{Nr} 842^{a}$. Hansteen und die Berge nördlich gezeichnet, nicht Melloni; nur ein ganz kleiner Crater etwas sudlich von Lohrmann's $\mathrm{Nr} .20$.

I 865 Juni 19. Abbd. Nr. yo4. Am Orte des Melloni zwei parallele schwache Hügel, $N-S$ gerichtet, kein Crater. Licht ungünstig. Es war um 15.9 Uhr bei abnehmender Phase.

I 865 Octob. I und $3 \mathbf{I}$. Beidemale war die Lichtgränze nahe westlich bei Melloni. Er ward nicht mit ver. zeichnet, was geschehen wäre, falls höhere Theile in der Nachtseite schon erleuchtet gewesen wären.

1865 Nov. 30. Abd. Lichtgränze in $62^{\circ}$ Ost. Abbd. Nr. I053. Dargestellt ist nur die Figur $H$, nördlich geöfnet, ahnlich wie $\mathrm{Nr} .904$.

1865 Dec, 29. Abbd. Nr. Io70. Hier ist (am Orte meines Melloni) ein wirklicher ringsgeschlossener Crater gezeichnet, aber nur nachlässig angelegt, weil die Luft ungünstig war, und weil nur ein schwächeres Ocular in Anwendung kam. Indem ich jetzt, wie oft, nur Situation zeichnete, ohne die Darstellung feineren Details zu erstreben, sah ich die Figur entweder wirklich geschlossen, oder zeichnete sie so, wissend wie jeder Beobachter, dass die meisten Crater hart an der Phase, nur unvollständig er. leuchtet erscheinen. Denn in den meisten Fällen, besonders aber bei sehr bekannten Formen, weiss man in Voraus, dass die Figur in Kurzem ganz erleuchtet sein wird, und zeichnet sie so zur bessern Charakteristik des Entwurfes der Landschaft. Anders verhält es sich freilich, wenn der Zustand der I uft Detailaufnahmen gestattet, und da soll man zeichnen, was man sieht, denn aus der stückweis sich vollendenden Beleuchtung eines Ringgebirges schliesst man auf die Gliederung der einzelnen Theile und auf die ungleichen Höhen. Indessen ist es wohl möglich, dass Dec. 29 die Figur $H$ im Norden wirklich geschlossen war durch einen Wall von ganz geringer Erhebung, der nur bei sehr geringer Sonnenhöhe erkannt wird. 
1866 Nov. I9. Phase in $60^{\circ}$ Ost. Abbd. Nr. I 154 . Genau am Orte von Lohrmanns $\mathrm{Nr} .20$ zeichnete ich die Figur $H$.

I 867 Febr. I6. Phase in $61^{\circ}$ Ost. Abbdi. Nr. I 265. Nur $H$ gezeichnet.

1873 April 9. Abbd. Nr. 1885. Nur $H$ gesehen und gezeichnet.

1875 Octub. II. Abbd. Nr. 2 roo. ebenso.

1875 Octob. I4. Im Vollmonde ist am Orte des Melloni Nichts sichtbar.

I 875 Nov. 10. Abbd. Nr. 2145 . Es ward nur $H$ gezeichnet.

1879 April 4. Abbd. Nr. 240I. Luft sehr schlecht. Weder Lohrmanns Crater noch $H$ ward gezeichnet, sondern nur einige schwache Hügelzige.

1881 März I2. Abbd. Nr. 2773. $H$ gezeichnet am Refr. von Reinfelder.

188, Mai 10. Abbd. Nr.2865. ebenso.

I881 Juni 9. Abbd. Nr. 2915. ebenso.

I88 I Juni II. Vollmond; am Orte zeigte sich nur eine sehr matte Aufhellung in der grauen Ebene.

Was zunächst Lohrmanns Darstellung betriftt, die wir als eine sehr sorgfältige erkennen, so darf man im Allgemeinen für möglich halten, dass damals wirklich ein starker Crater am angezeigten Orte existirte. Auch der Crater Linné, der ungeachtet seines geringen Durch. messers von Mädler als Fixpunkt erster Ordnung für die selenographischen Ortsbestimmungen benutzt ward, ist der Art verschwunden, oder vielmehr in seiner Sichtbarkeit verringert, dass er seit vielen Jahren sich, wenn für ihn die Sonne auf oder untergeht, nur als der feinste Hügelpunkt am starken Fernrohre zeigt; bei höherer Beleuchtung aber wohl eine weisse Wolke; durchaus aber nicht mehr die wirkliche Craterfigur darstellt, wie sie einst von Lohrmann, von Mädler und mir gesehen ward.
Es ist aber auch nicht ganz unwahrscheinlich, dass es Lohrmann wie mir selbst erging, dass bei dem Entwurf der Charte das Original undeutlich war, oder nicht richtig gedeutet ward. Indem er den Halbcrater $H$ als solchen nicht deutlich sah, ihn aber, was geschehen kann, für einen vollständigen Crater hielt, und im Originale etwas zu stark ausprägte, brachte er ihn auf die definitive Zeichnung, die nun der Wirklichkeit nicht genuigend entspricht.

Aus meinen Beobachtungen folgt, dass von I 865 bis I88 I sich am Orte von Lohrmann Nr. $20=$ Melloni $=\mathrm{Nr} .3$ bei mir, kein ansehnlicher Crater, sondern nur die flache Figur $H$ befunden habe.

Ich schliesse nun, nach meiner Ueberzeugung mit Sicherheit, dass ich während des Entwurfes meiner Sect. 19, auf die eine Abbildung Nr. I070 (I 865 Dec. 29) zu grosses Gewicht gelegt habe, und wohl besonders desshalb, weil ich, ursprünglich die Situation nach Lohrmanns Charte im Grossen anlegend, auf Lohrmanns Crater mehr als zulässig Rücksicht nahm, dabei aber doch die Figur $H$ nahezu an den richtigen Ort stellte, ohne damals die Angelegenheit mit der jetzigen Sachkenntniss hinreichend prüfen zu können.

Indem ich nun den Namen Melloni bei der Figur $H$ belasse, erkläre ich den grossen Crater Nr. 3 in meiner Sect. 19 als einen blossen Redactionsfehler, und ersuche die Besitzer der. Charte, den Crater $\mathrm{Nr}, 3$ als nicht existirend $z u$ durchstreichen.

Ich habe diese Mittheilung für wünschenswerth gehalten, und hofie dadurch das Auftauchen neuer Fabeln uiber angebliche Veränderungen auf dem Monde noch rechtzeitig zu verhindern.

Athen $188 \mathrm{I}$, Juni 12.

\section{Definitive Bahn-Elemente des Cometen 1877 I (Borrelly-Pechiile).}

Der Comet I877 I wurde von Herrn Borrelly in Marseille am 8. Februar und davon unabhängig von Herrn Pechüle in Kopenhagen am 9. Febr. entdeckt und bis zum 3. April beobachtet. Da letzterer Astronom an der von ihm beabsichtigten definitiven Bahnbestimmung des
Cometen, welchen er am längsten verfolgt hat, verhindert ist, habe ich dieselbe übernommen.

Zunächst habe ich aus den 3 Beobachtungen, welche mittelst der vorhandenen vorläufigen Elemente für $\mathrm{Pa}$ rallaxe und Aberration verbessert wurden,
I 877
I) Februar 10
2) $\$ 28$
3) März I 7

$$
\begin{aligned}
& a \text { app. } \\
& 17^{\mathrm{h}} 18^{\mathrm{m}} 58^{\mathrm{s}} 7 \\
& 3839.68 \\
& 422 \text { I. } 42 \\
& \begin{array}{c}
\text { Japp. } \\
+\quad 5^{0284} 43^{\prime o} \\
+\quad 6811 \text { 1.8 } \\
+505642.6
\end{array}
\end{aligned}
$$

auf den Aequator auf die Ekliptik

$T=1877$ Januar $19.219285 \mathrm{~m}$. Zt. Berlin

$\pi=174^{\circ} 25^{\prime} 56^{\prime \prime} 2$ O

$88=187$ … 1524.34 Mittl. Aequinox 1877.0

$i=152 \quad 5453.45$

$\log q=9.907090$.
$T$ I 1877 Januar I9.219285 m. Zt. Berlin

$\left.x^{\prime}=-167^{\circ} 42^{\prime} 52^{\prime \prime} 6\right)$

$\Omega^{\prime}=$ I 84 I6 43.3$\}$ m. Aeq. 1877.0.

$i^{\prime}=.129 \quad 34 \quad 2.6$

$\log q=9.907090$ 